\title{
DISTRIBUCIÓN Y ABUNDANCIA DE LA POBLACIÓN REPRODUCTORA DE TREPARRISCOS (TICHODROMA MURARIA) EN ARAGÓN
}

\section{Distribution and abundance of the breeding population of Wallcreeper (Tichodroma muraria) in Aragon}

\author{
Juan Antonio Gil ${ }^{1,2, *}$ y Carlos Pérez ${ }^{2}$ \\ ${ }^{1}$ Fundación para la Conservación del Quebrantahuesos (FCQ), Plaza San Pedro Nolasco 1, 4-F. 50001 Zaragoza. \\ Tlf./Fax: 9762996 67.fcq@quebrantahuesos.org \\ Más información: www.quebrantahuesos.org \\ ${ }^{2}$ Grupo Aragón de Anillamiento Científico de Aves (GAA), Estación Zaragoza-Delicias Módulo 5. C/ Rioja, 33.50011 Zaragoza. \\ grupoaragonanillamiento@gmail.com \\ Más información: www.grupoaragondeanillamiento.es \\ *Autor corresponsal
}

Recibido: 03-02-2015. Aceptado: 19-03-2015. Fecha de publicación on-line: 10-09-2015

Citation / Cómo citar este artículo: Gil, J.A. \& Pérez, C. (2015). Distribución y abundancia de la población reproductora de Treparriscos (Tichodroma muraria) en Aragón. Pirineos, 170, e011. doi: http://dx.doi.org/10.3989/Pirineos.2015.170004

RESUMEN: Con la realización del Atlas Ornitológico de Aragón tan solo se efectuó una primera aproximación de la distribución de la población reproductora del treparriscos (Tichodroma muraria) en Aragón. En el presente artículo se ha actualizado la información sobre su distribución y se ha realizado una primera estima de su población. En total se obtuvieron 145 registros de la época de reproducción (mayo-agosto) entre los años 1982-2014. Los sectores de cría se sitúan a una media de $2.409 \pm 572$ m.s.n.m. El 62\% de los registros se realizan en áreas con rocas básicas. La construcción del nido se realiza entre mayo y principios de junio, la incubación entre junio y julio, los pollos están en el nido entre junio y finales de julio y los grupos familiares se forman en agosto. La distribución ocupa un total de 40 cuadrículas UTM de 10x10 Km (un aumento del 133\% sobre la distribución dada en el Atlas) y 87 cuadrículas $1 x 1 \mathrm{~km}$. Está presente en todos los macizos montañosos del Pirineo aragonés, a excepción de Culfreda, Acherito y Mesa de los Tres Reyes, donde no se obtienen registros de su presencia. El área de ocupación con presencia comprobada es de $83 \mathrm{~km}^{2}$ y de ocupación potencial es de $664,96 \mathrm{~km}^{2}$ y un área de máxima ocupación de 495,45 km². Se ha estimado una población de 705 territorios reproductores para el área potencial, con una densidad de territorios de 0,13 territorios $/ \mathrm{km}^{2}$ y de 525 territorios para el área de máxima ocupación, con una densidad de 0,18 territorios $/ \mathrm{km}^{2}$. Con la información disponible de la especie en Aragón (1.410 individuos reproductores), el treparriscos debería estar incluida en la categoría de Preocupación Menor (criterios UICN) y debería realizarse una actualización de su estima de población en Pirineos (vertiente Sur) y resto de España.

PALABRAS CLAVE: Treparriscos, Tichodroma muraria, distribución, población, Pirineo, Aragón.

ABSTRACT: With the completion of the Ornithological Atlas of Aragon only a first approximation of the distribution of the breeding population of Wallcreeper (Tichodroma muraria) in Aragon was made. In this paper we update 
the information about its distribution and we made a first estimate of population size. Overall total 145 records were obtained during the breeding season (May-August) between the years 1982 to 2014 are obtained. Breeding areas were located at an average altitude of $2.409 \pm 572$ meters, and $62 \%$ records were made in areas with basic rocks. Nest building took place between May and early June, incubation between June and July, chicks rearing between June and late July and family groups were formed in August. The distribution encompasses 40 UTM 10x10 km (an increase of $133 \%$ on the distribution given in the Ornithological Atlas of Aragon) and $871 \mathrm{x} 1 \mathrm{Km}$ squares. It is present in all the Aragon Pyrenees mountain ranges, except for Culfreda and Acherito and Mesa de los Tres Reyes, where no records of presence were obtained. The area of occupancy with proven presence was $83 \mathrm{Km}^{2}$ and potential occupancy areas was $664.96 \mathrm{Km}^{2}$ and an area of maximum occupancy of $495.45 \mathrm{Km}^{2}$. We estimated population size on 705 breeding territories potential for the area, territories with a density of 0.13 territories $/ \mathrm{Km}^{2}$ and 525 territories for the area of maximum occupancy, with a density of 0.18 territories $/ \mathrm{Km}^{2}$ Taking into account existing information available on the species in Aragón (1,410 breeding individuals), the Wallcreeper should be included in the category of Least Concern according (IUCN criteria) and its population size should be updated in the Pyrenees (southern slope).

KEYWORDS: Wallcreeper, Tichodroma muraria, distribution, population, Pyrenees, Aragon.

\section{Introducción}

La dinámica de las poblaciones de paseriformes residentes en los ecosistemas alpinos es poco conocida, a pesar de que dichos ecosistemas y en consecuencia la comunidad de especies que en ellos habita son especialmente vulnerables a los efectos del cambio climático. Según Araújo et al. (2011) la mayor parte de las especies de vertebrados terrestres de la Península Ibérica sufrirán contracciones significativas de sus distribuciones climáticas durante este siglo. El treparriscos (Tichodroma muraria) es un paseriforme muy representativo de los hábitats alpinos, especializado en la explotación de paredes de roca (Lörhrl \& Wilson, 2008), que posee una distribución paleomontana. La escasez de información y conocimiento se debe, en gran medida, a la dificultad de su estudio. Durante la época de reproducción el área de distribución de la especie se extiende por la Cordillera Cantábrica, Pirineos, Alpes, Apeninos, Cárpatos, la alta montaña de Grecia y Turquía y en el Cáucaso, montañas de Kopet-Dag (Turkmenistán), montañas del este y norte de Irán (Kerman, Khorasan, Zagros, Elburz), el Tien Shan y Trans-Ili Alatau (Kazajstán), a Kirguizia, Tayikistán, el macizo del Himalaya, desde Afganistán y Pakistán al Tibet, India y Nepal, los montes Altai (Mongolia) y el extremo oeste de China, sobre todo cerca del Himalaya y en la meseta de Quighai-Xizang hasta Yunnan (Glutz von Blotzheim et al., 1993; Cramp \& Perrins, 1993). En España las áreas de cría se sitúan en la Cordillera Cantábrica: Picos de Europa, SO de Asturias, NE de León y NO de Palencia y Pirineos: Navarra (valle del Roncal-Larra), Aragón (Huesca) y Cataluña (Lérida y Gerona) (Elosegui, 1985; Báguena et al., 1998; Herrero, 2003; Santandreu et al., 2004). En Aragón se distribuye desde Peña Ezcaurre (valle de Ansó), hasta la Alta Ribagorza (Llauset), localizándose el enclave más meridional en el macizo del Turbón (Báguena et al., 1998). Durante el invierno también está presente en el valle del Ebro y el Sistema Ibérico (Zaragoza y Teruel). En España se realizó una primera estima de su población durante la ejecución del
Atlas de las Aves de España (1975-1995): 9000-12.000 parejas (Hernández, 1997), que posteriormente fue utilizada para estimar la población europea: 14.341-19.542 parejas (Hagemeijer \& Blair, 1997). En el año 2000 se volvió a estimar la población europea, arrojando una cifra de 38.000-100.000 parejas (Lörhrl \& Wilson, 2008). Los datos disponibles indican que las primeras estimaciones están sobreestimadas. Basándose en el área de distribución conocida, los censos parciales detallados y las densidades habituales de la especie, probablemente las poblaciones españolas estén entre 500 y 1.000 parejas (Aymerich et al., 2012). Sanz-Zuasti \& Velasco (1999) estiman las poblaciones de Castilla y León en un máximo de 600 parejas. En el Pirineo catalán se efectuó una primera estima de población durante la realización del Atlas de las aves nidificantes (1999-2002): 40-100 parejas (Santandreu et al., 2004), que posteriormente fue revisada, estimándose un mínimo de 65 parejas (Aymerich et al., 2012). Con la realización del Atlas Ornitológico de Aragón se efectúa la primera aproximación sobre la distribución de la población reproductora aragonesa, que está presente en 30 cuadrículas de 10x10 Km (Báguena et al., 1998). Sin embargo, no se realizó ninguna estima de población, a pesar de existir trabajos sobre cálculos de densidades: 0,19 aves/10 ha en la Brecha de Roland (Valle de Ordesa), 0,28 aves/10 ha en Ballibierna (valle de Benasque), 0,65 aves/10 ha en Lecherines (valle de Canfranc) (Antor, 2002), y 9-11 parejas/ $\mathrm{Km}^{2}$ (Pedrocchi et al., 2007). Tan solo existe una estima de población para el Parque Nacional de Ordesa y Monte Perdido (PNOMP) de 40-50 parejas (Woutersen \& Grasa, 2002). El conocimiento del número de territorios y su distribución en el Pirineo aragonés se hace imprescindible para poder estimar adecuadamente la población pirenaica, su estado de conservación y evolución. El objetivo prioritario de este artículo es revisar, mejorar y ampliar la información sobre la población y distribución del treparriscos en Aragón, con el fin de poder disponer de una cartografía temática que muestre la distribución espacial de la especie, así como poder estimar su población. 


\section{Material y métodos}

\section{1. Área de estudio}

El trabajo se desarrolló en la vertiente sur del Pirineo central (Huesca/Aragón/ España), comarcas de la Jacetania, Alto Gállego, Sobrarbe y la Ribagorza $(7.885,62$ $\mathrm{Km}^{2}$ ). Zona montañosa perteneciente a la región biogeográfica Eurosiberiana. Limita al norte con Francia, al sur con la depresión del Ebro (anchura 55-75 Km), al oeste con Navarra y al este con el río Noguera-Ribagorzana (Lérida) (longitud $135 \mathrm{Km}$ ). El Pirineo forma parte de las Cordilleras que se levantaron durante la orogenia alpina hace 80-85 millones de años. El Pirineo Axial está constituido por rocas paleozoicas (ígneas y metamórficas) y un conjunto de alineaciones cuya cima más alta es la del Aneto (3.404 m.s.n.m.) (Foto 1), mientras que las Sierras Interiores están formadas por afloramientos mesocenozoicos (Foto 2). El Pirineo Axial posee macizos con batolitos, que aparecen rodeados por rocas metamórficas antiguas, con predominio de calizas y pizarras carboníferas o devónicas en el sector occidental y de cuarcitas, pizarras y esquistos del Devónico al Cámbrico en el oriental: Balaitús-Infierno-Argualas, Posets-Eriste, Maladeta-Aneto. Estos macizos presentan un relieve de escarpadas y agudas crestas graníticas. Al sur del Pirineo Axial aparecen las Sierras Interiores: Bisaurín, Collarada, Monte Perdido (3.355 m.s.n.m.), etc., con crestas y torreones piramidales armados en las calizas cretácicas o eocenas. La Depresión Media, situada entre las Sierras Exteriores y las Interiores, está formada por un relleno de sedimentos blandos, destaca la Canal de Berdún y las sierras de San Juan de la Peña, Oroel y Canciás. Las Sierras Exteriores están ubicadas en el extremo meridional y están constituidas por calizas, margas y conglomerados, destacando las sierras de Guara y Santo Domingo. El clima corresponde al me-

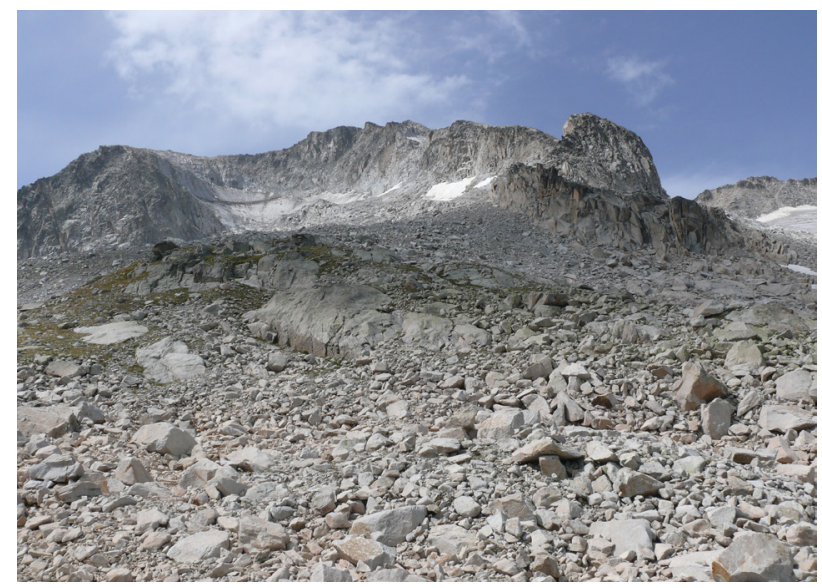

Foto 1: Área granítica, correspondiente al sector oriental de la comarca de la Ribagorza (macizo de la Maladeta) (autor: J.A. Gil/FCQ).

Photo 1: Granitic area, corresponding to the eastern part of the region of Ribagorza (Maladeta) (author: J.A. Gil / FCQ). diterráneo montano, de carácter continental, repartiéndose la vegetación en varios pisos bioclimáticos: piso colino (situado entre los 500-800 m.s.n.m. y dominado por una vegetación submediterránea), piso montano (situado entre los 800-1.600 m.s.n.m. y dominado por una vegetación de montaña media centroeuropea, compuesta por pinares de pino silvestre (Pinus sylvestris), hayedos (Fagus sylvatica), abetales (Abies alba) y bosques mixtos), piso subalpino (situado entre los 1.600-2.300 m.s.n.m., compuesto por amplias praderas alpinas y por pinares de pino negro (Pinus uncinata), con sotobosque de rododendro (Rhododendron ferrugineum) y arándano común (Vaccinium myrtillus), como especies más representativas) y piso alpino (situado entre los 2.300-3.000 m.s.n.m.; constituye el último piso de esta gradación y está tapizado por plantas herbáceas y extensas superficies de roca). La zona de presencia de treparriscos reproductores se localiza en el Pirineo Axial y las Sierras Interiores, concretamente en las áreas rocosas de los pisos subalpino y alpino (2.0003.000 m.s.n.m.) (Báguena et al., 1998). En el Pirineo aragonés los ambientes supraforestales (pastos, roquedos, gleras, etc.) de 1.800 hasta los 3.000 m.s.n.m. ocupan una superficie de $1.491,58 \mathrm{Km}^{2}$, de los cuales $44,5 \%$ corresponden a hábitats rupícolas con una pendiente por encima de $\operatorname{los} 65^{\circ}$ (roquedos, gleras, bloques, etc.), que son los utilizados por la especie para la reproducción. El 50\% de estos macizos poseen rocas ácidas con alto contenido en sílice $(>60 \%)$, el $40 \%$ rocas básicas $(<55 \%$ de sílice) y el $10 \%$ rocas intermedias $(55-60 \%)$.

\subsection{Fuentes de información}

La primera vez que se cita el treparriscos en Aragón fue por Asso (1784) y posteriormente en el valle de Ordesa por Wallis (1895). Para la elaboración de este artículo se han recopilado observaciones de diferentes fuentes:

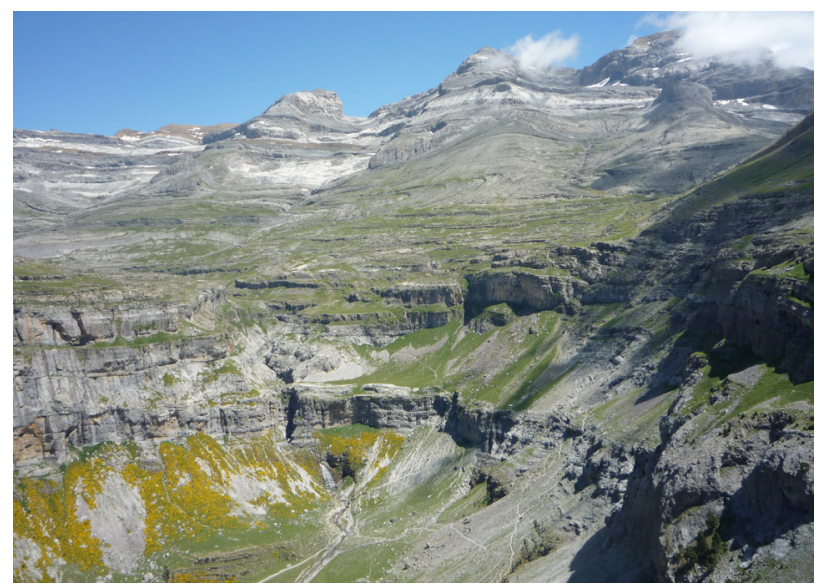

Foto 2: Área cálcarea, correspondiente al sector central de la comarca de Sobrarbe (macizo del Mte. Perdido) (autor: J.A. Gil/FCQ).

Photo 2: Calcareous area corresponding to the central sector of the Sobrarbe (Massif Lost Mtn.) (Author: J.A. Gil / FCQ). 
datos propios (prospecciones específicas de los autores del artículo y de miembros de la Fundación para la Conservación del Quebrantahuesos -FCQ-), Atlas Ornitológico de Aragón 1987-1991, Anuario Ornitológico de Aragón «Rocín» 1991-2011 (http://anuariorocin.blogspot. com.es/), Grupo Ornitológico Oscense (GO) (http://www. avesdehuesca.es/), Anuario Ornitológico de Navarra 1995 (https://sites.google.com/site/scngorosti/Home/novedades-de-la-sociedad/elanuarioornitologicodenavarradigital) y Anuario Ornitológico de Cataluña 2006-2007 (http://www.ornitologia.org/ca/queoferim/divulgacio/publicacions/anuari_pdf.html), que han ido completando la base de datos de la FCQ. Cada observación está referenciada en coordenadas Universal Trasverse Mercator (UTM), con la información del observador, fecha, lugar, observaciones e intervalos altitudinales. Se han considerado las observaciones del periodo reproductor comprendido entre mayo y agosto. Este periodo se ha seleccionado por la información existente sobre la fenología de la reproducción de la especie en Aragón, recopilada durante los últimos 32 años y las referencias bibliográficas existentes (Hernández et al., 1993a; Lörhrl \& Wilson, 2008; Aymerich et al., 2012). Para el tratamiento de los datos georreferenciados se han utilizado herramientas SIG (Sistemas de Información Geográfica) Arc GIS 9.1 (ESRI, 2005), con la que se ha generado la cartografía temática. Todas las capas de información geográfica, vectorial, fueron georreferenciadas y proyectadas en coordenadas métricas UTM con origen en el huso 30. Para el tratamiento del tipo de rocas: ácidas, intermedias y básicas, se utilizó la cobertura del Instituto Geológico y Minero de España (IGME): http://info.igme.es/.

\subsection{Distribución}

La distribución que se presenta se ha elaborado con los datos recopilados entre 1982-2014, utilizándose cuatro tipos de descripciones:

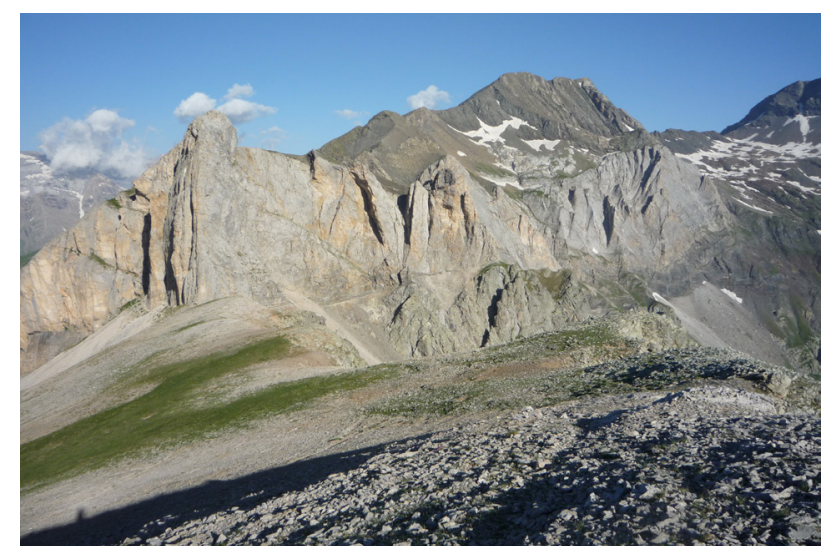

Foto 3: Sierra de Liena y macizo de la Munia, situado en el sector central de la comarca de Sobrarbe (autor: J.A. Gil/FCQ).

Photo 3: Sierra de Liena and Munia massif, located in the central sector of the Sobrarbe (author: J.A. Gil / FCQ)
1. Sectores geográficos: occidental (comarca de la Jacetania: $1.857,83 \mathrm{Km}^{2}$ ), central (comarcas de Alto Gállego: 1.360,35 Km² y Sobrarbe: 2.203,97 Km²) (Fotos 3 y 4) y oriental (comarca de la Ribagorza: $2.463,47 \mathrm{Km}^{2}$ ). El sector con mayor superficie de áreas rocosas $\left(65^{\circ}\right.$ de pendiente por encima de los 2.000 m.s.n.m., con roquedos, gleras, bloques) es Sobrarbe $\left(551,49 \mathrm{Km}^{2}\right)$, así como de zonas supra forestales $\left(545,44 \mathrm{Km}^{2}\right)$, pero proporcionalmente es Ribagorza la comarca que posee mayor superficie de áreas rocosas $(49,46 \%)$, siendo las comarcas de Alto Gállego (sector central) y Ribagorza (sector oriental) las que presentan mayor porcentaje de áreas rocosas ácidas $18,42 \%$ y $23,86 \%$, respectivamente.

2. Espacios Naturales Protegidos (ENP): Los Valles Occidentales-Jacetania $\left(\mathrm{n}=271,16 \mathrm{~km}^{2}\right)$ con un $39,35 \%$ de áreas rocosas, PNOMP $\left(156,92 \mathrm{Km}^{2}\right)$ con un $56,13 \%$ de áreas rocosas y Parque Natural Posets-Maladeta-Ribagorza $\left(334,76 \mathrm{Km}^{2}\right)$ con un $63,44 \%$ de áreas rocosas.

3. Mapas de distribución en cuadrículas de 10x10 km $\left(100 \mathrm{Km}^{2}\right)$, distinguiéndose en cada cuadrícula la información sobre la reproducción posible, probable y segura según los criterios del European Ornithological Atlas Commitee (-EOAC-, Hagemeijer \& Blair, 1997) y en cuadrículas $1 \mathrm{x} 1 \mathrm{~km}$.

4. Principales macizos montañosos: Maladeta-Aneto, Posets-Eriste, Clarabide-Perdiguero-Boum, sierra Llauset-Negra-Castanesa, Turbón, Cotiella, Monte Perdido, La Munia, Culfreda, Punta Suelza, Bachimala, Vigñemale, sierra Tendeñera-Telera, BalaitúsInfierno-Argualas, Collarada, Anayet, Aspe, Bisaurín, Peña Forca, Acherito y Mesa de los Tres Reyes.

\section{4. Área de ocupación}

Para estimar el área de ocupación de la especie y su área potencial, se han empleado las observaciones de la

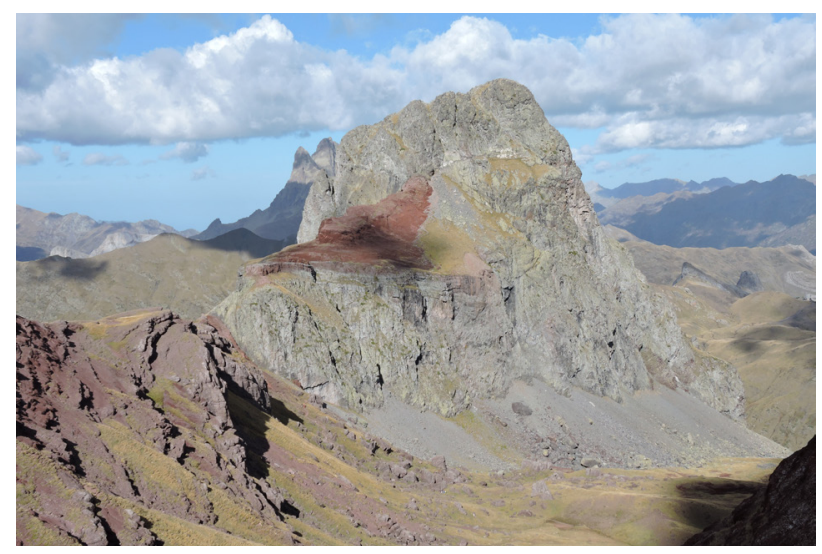

Foto 4: Macizo del Anayet, situado en el sector central de la comarca del Alto Gállego (autor: J.A. Gil/FCQ).

Photo 4: Anayet Massif, located in the central sector of the Alto Gallego (author: J.A. Gil / FCQ). 
época de reproducción y se han trasladado a cuadrículas 1x1 Km. Estos datos son superpuestos sobre el área de rocas, bloques y gleras, con un $65^{\circ}$ de pendiente por encima de los 2.000 m.s.n.m. de altitud, se localizan el $85 \%$ de las parejas reales entre los rangos de altitud de (2.0003.000 m.s.n.m.). Esto nos permite delimitar un área de ocupación cartográfica, con el hábitat potencial utilizado por la especie. Para aproximarnos más a la población real, se ha estimado el área de máxima ocupación, para ello, se ha calculado sobre el rango de cota de altitud donde se localizan más del 71\% de las parejas reales en el Pirineo aragonés y que se encuentra situado por encima de 2.300 m.s.n.m., el rango utilizado es de 2.300-3.000 m.s.n.m. Otros autores, Hernández et al., (1993b), determinan la altitud media en los 1.929 m.s.n.m. para los Pirineos, mientras que en el PNOMP, Woutersen \& Grasa (2002) estiman como el sector óptimo para la especie un intervalo altitudinal de entre 1.900-2.920 m.s.n.m, obteniendo una media de 2.400 m.s.n.m.

\subsection{Estimación de población}

La estima del tamaño poblacional se realizó a partir del número de territorios con presencia comprobada en Aragón durante la época de reproducción en el periodo comprendido entre 1982 y 2014, asumiendo que el treparriscos es fiel a las localidades de cría (Saniga, 2004; Lörhrl \& Wilson, 2008) y la disponibilidad de hábitat potencial. Esto queda bien demostrado con algunas de las parejas del área de estudio, que cuentan con una dilatada antigüedad ocupando el mismo territorio: Gruta de Casteret (macizo de Monte Perdido) con datos que van desde 1986 hasta 2009, o la pareja localizada en el Congosto de Ventamillo (macizo de Cotiella) en la que se registran datos desde el año 1983 hasta el año 2004. Se consideró como territorio reproductor aquel con presencia constatada de la especie entre mayo y agosto. Para distinguir los diferentes territorios en base a las observaciones de individuos de las parejas contiguas, se ha considerado que eran territorios diferentes cuando existía hábitat potencial y las observaciones estaban separadas más de $500 \mathrm{~m}$. Este criterio se ha adoptado según la extensión lineal de los territorios (Lörhrl \& Wilson, 2008; Saniga, 2004) y la extensión de los territorios estimados en el Pirineo catalán: en rocas calizas tienen un tamaño medio de $0,12 \mathrm{Km}^{2} \mathrm{y}$ en zonas graníticas tienen un tamaño medio de $0,59 \mathrm{~km}^{2}$ (Aymerich et al., 2012).

\subsection{Densidad}

Se ha estimado la densidad para el área potencial (número de territorios comprobados/área de ocupación potencial), área de máxima ocupación (número de territorios comprobados/área de máxima ocupación potencial), que poseen hábitats favorables (áreas rocosas) y el área supraforestal (número de territorios comprobados/área supraforestal) en donde se incluyen los pastizales. Se ha analizado de igual manera áreas más reducidas a escala local, cogiendo como modelo los ENP.

\subsection{Estado de conservación}

Para conocer el estado de conservación del treparriscos se han utilizado las categorías y criterios de la Unión Internacional para la Naturaleza (UICN, 2012). A partir de la información de los datos de la población y del área de ocupación se ha designado su categoría de conservación. La población se define como el número total de individuos que pueden reproducirse y el área de ocupación como la zona dentro de la extensión de presencia.

\section{Resultados}

\subsection{Fenología de la reproducción y hábitat}

Se ha recopilado un total de 145 observaciones en la época reproductora del treparriscos, en el período 19822014. El 70\% de los registros corresponden a observaciones del período 1992-2014. De las 145 observaciones, 79 son reproducciones posibles, 16 probables y $50 \mathrm{segu}-$ ras. Se registraron las fechas más tempranas y más tardías de construcción del nido, incubación, pollos en nido y grupos familiares (Tabla 1). El 55\% de las observaciones se realizaron en ENP, de éstas el $45 \%$ se realizaron en el PNOMP. Los sectores de cría se situaron a una altitud media de $2.397 \pm 518$ m.s.n.m. (rango 800-3.200; $\mathrm{n}=$ 62). Mientras que en el sector oriental (Posets-Maladeta) y central (PNOMP) la media de las áreas de cría se situaron a $2.662 \pm 321$ m.s.n.m. (rango 2.300-3.200; $\mathrm{n}=9$ ) y $2.602 \pm 294$ m.s.n.m. (rango 1.900-3.000 m.s.n.m.; $\mathrm{n}=$ 18). En el sector occidental se situaron a $2.150 \pm 506$ m.s.n.m. (rango 1.050-2.600 m.s.n.m.; n =9). El 62\% de los registros se realizaron en áreas con rocas básicas (Tabla 2).

Tabla 1: Observaciones de construcción del nido, incubación, puestas, pollos en nido, y grupos familiares. Table 1: Observations of nest building, incubation, sunsets, chicks in nest, and family groups.

\begin{tabular}{|l|c|c|c|}
\hline & Fecha más temprana & Fecha más tardía & Nobservaciones $^{\mathbf{0}}$ ob \\
\hline Nido en construcción & 8 de mayo (1.900 m.s.n.m.) & 2 de junio (2.600 m.s.n.m.) & 4 \\
\hline Incubación & 17 de junio (2.800 m.s.n.m.) & 10 de julio (2.800 m.s.n.m.) & 2 \\
\hline Pollos en nido & 28 de mayo (2.700 m.s.n.m.) & 31 de julio (800 m.s.n.m.) & 30 \\
\hline Grupos familiares & 5 de agosto & 21 de agosto (2.600 m.s.n.m.) & 8 \\
\hline
\end{tabular}


6•J.A. GIL \& C. PÉREZ

Tabla 2: Observaciones de treparriscos según el tipo de rocas y los criterios de reproducción (Hagemeijer \& Blair, 1997). Table 2: Observations Wallcreeper by type of rocks and criteria reproduction (Hagemeijer \& Blair, 1997).

\begin{tabular}{|l|c|c|c|c|}
\hline & Posible & Probable & Segura & Total \\
\hline Cortados rocosos básicos & 39 & 13 & 38 & 90 \\
\hline Cortados rocosos ácidos & 25 & 0 & 10 & 35 \\
\hline Cortados rocosos mixtos & 7 & 2 & 1 & 10 \\
\hline Sin áreas rocosas & 8 & 1 & 1 & 10 \\
\hline Total & $79(54,5 \%)$ & $16(11 \%)$ & $50(34,5 \%)$ & 145 \\
\hline
\end{tabular}

\subsection{Distribución}

El treparriscos en la época reproductora está presente en los tres sectores en los que se dividió el Pirineo aragonés (occidental, central y oriental) y en cuatro comarcas

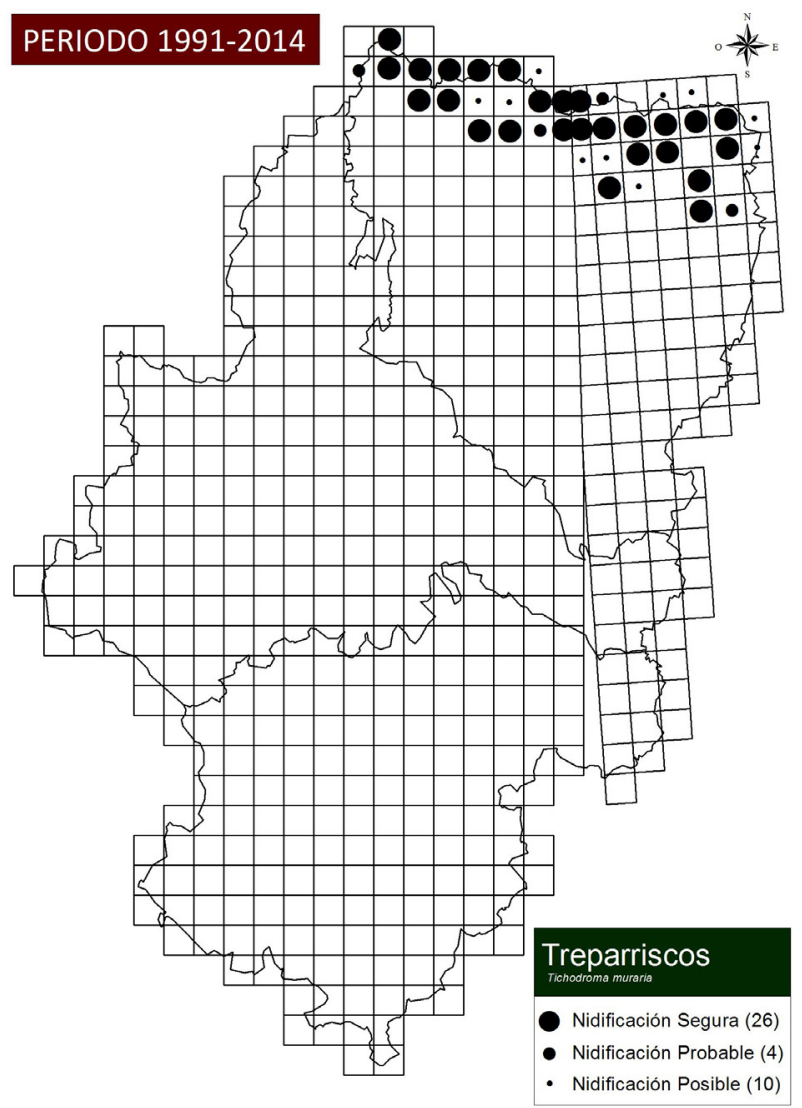

Figura 1: Mapa de distribución del treparriscos (Tichodroma muraria) en Aragón, cuadrículas 10x10 km, que incluye los datos del Atlas Ornitológico de Aragón (Báguena et al., 1998), más la incorporación de los registros obtenidos entre 1991 y

2014 y la información de los criterios de reproducción (Hagemeijer \& Blair, 1997).

Figure 1: Distribution map of Wallcreeper in Aragon, $10 x 10 \mathrm{~km}$ grid, which includes data Ornithological Atlas of Aragon (Báguena et al., 1998), incorporating more recordings between 1992 and 2014 and information criteria reproduction (Hagemeijer \& Blair, 1997).
(Jacetania, Alto Gállego, Sobrarbe y Ribagorza). La distribución actual abarca un total de 40 cuadrículas UTM de 10x10 Km, que incluye los datos del Atlas Ornitológico de Aragón (Báguena et al., 1998), más la incorporación de los registros obtenidos entre 1991 y 2014 y la información de los criterios de reproducción (Hagemeijer \& Blair, 1997) (Figuras 1 y 2). Una distribución real más detallada en base a las cuadrículas 1x1 Km (Figura 3 y Tabla 3) muestra que la especie ocupa de Oeste a Este

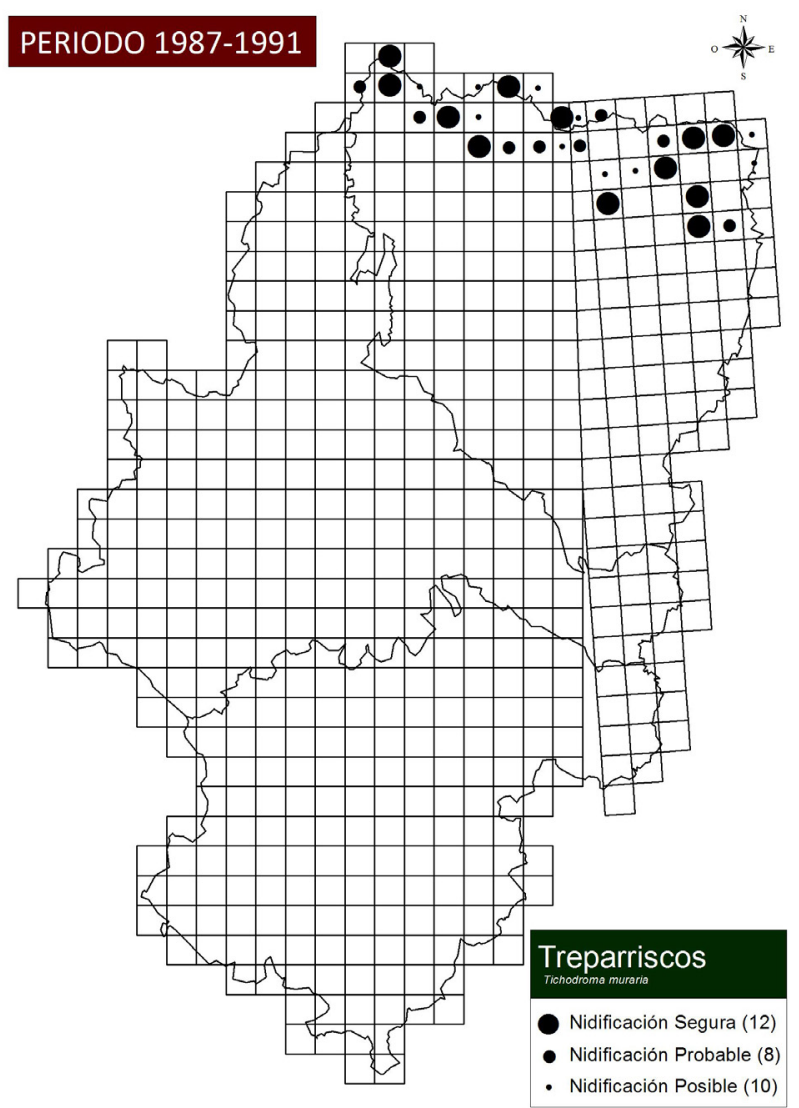

Figura 2: Mapa de distribución del treparriscos (Tichodroma muraria) en Aragón, cuadrículas $10 \times 10 \mathrm{~km}$, con los datos del Atlas Ornitológico de Aragón 1987-1991 (Báguena et al., 1998).

Figure 2: Distribution map of Wallcreeper in Aragon, $10 \times 10 \mathrm{~km}$ grid with data Ornithological Atlas of Aragon 1987-1991 (Báguena et al., 1998). 


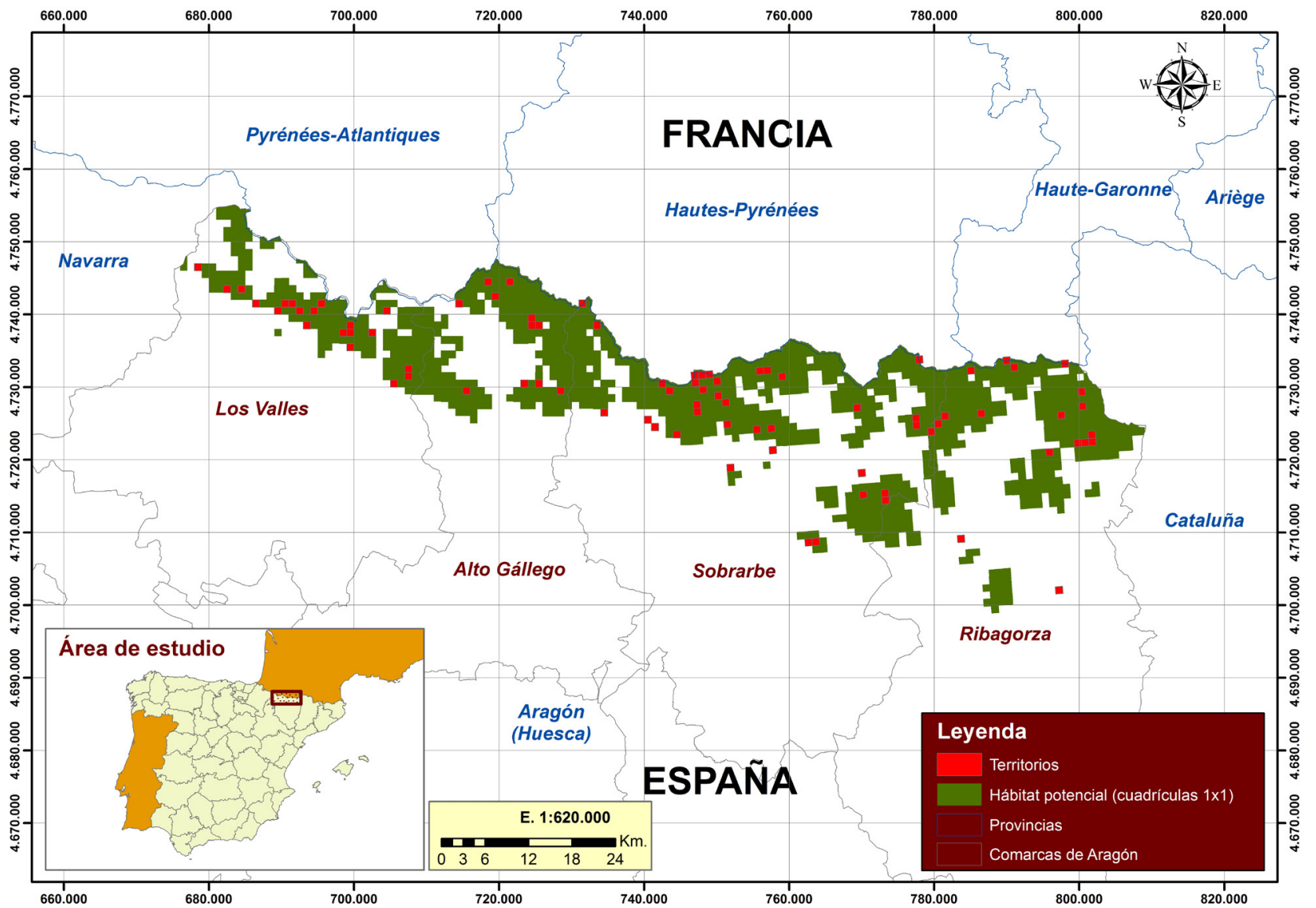

Figura 3: Mapa del área de ocupación con presencia comprobada y potencial del treparriscos (Tichodroma muraria) en Aragón.

Figure 3: Map of the area occupied with proven presence and potential Wallcreeper in Aragon.

todo el Pirineo aragonés, con presencia en 87 cuadrículas. La especie está presente en todos los macizos montañosos a excepción de Culfreda, Acherito y Mesa de los Tres Reyes, donde no se obtienen registros de su presencia.

\section{3. Área de ocupación}

El área de ocupación con presencia comprobada de la especie es de $83 \mathrm{~km}^{2}$. El área de ocupación potencial es de $664,96 \mathrm{~km}^{2}$ (Figura 4) como resultado de las observaciones

Tabla 3: Sectores del Pirineo aragonés con presencia de treparriscos, según cuadrículas $1 \times 1 \mathrm{Km}$ e información sobre los criterios de reproducción (Hagemeijer \& Blair, 1997). Table 3: Area of the Aragonese Pyrenees presence of Wallcreeper as $1 \times 1 \mathrm{Km}$ and information on criteria for reproduction (Hagemeijer \& Blair, 1997).

\begin{tabular}{|l|c|c|c|c|}
\hline & Posible & Probable & Segura & Total \\
\hline S. occidental & 27 & 1 & 18 & 46 \\
\hline S. central & 14 & 1 & 5 & 20 \\
\hline S. oriental & 9 & 2 & 10 & 21 \\
\hline Total & $50(57,5 \%)$ & $4(4,5 \%)$ & $33(38 \%)$ & 87 \\
\hline
\end{tabular}

comprobadas y la superficie de áreas rocosas de $65^{\circ}$ de pendiente por encima de los 2.000 m.s.n.m. de altitud y de 495,45 para el área de máxima ocupación (Figura 5), donde la cota altitudinal se encuentra por encima de los 2.300 m.s.n.m.

\subsection{Estimación de población}

El número de territorios con presencia comprobada en Aragón fue de 88, localizándose el 23,9\% en el sector occidental (Jacetania) (21 territorios), el 56,8\% en el sector central (Álto Gállego y Sobrarbe) (10 y 40 territorios, respectivamente) y $19,3 \%$ en el sector oriental (Ribagorza) (17 territorios), siendo el macizo del Monte Perdido el que más territorios posee $(\mathrm{n}=23)$. Mediante este número de territorios comprobados y la disponibilidad potencial de hábitat, se ha estimado una población de 525 territorios para el área de máxima ocupación y de 705 territorios para el área potencial del Pirineo aragonés.

\subsection{Densidad}

La densidad de territorios estimada en el área potencial es superior a la del área supra forestal e inferior a la 


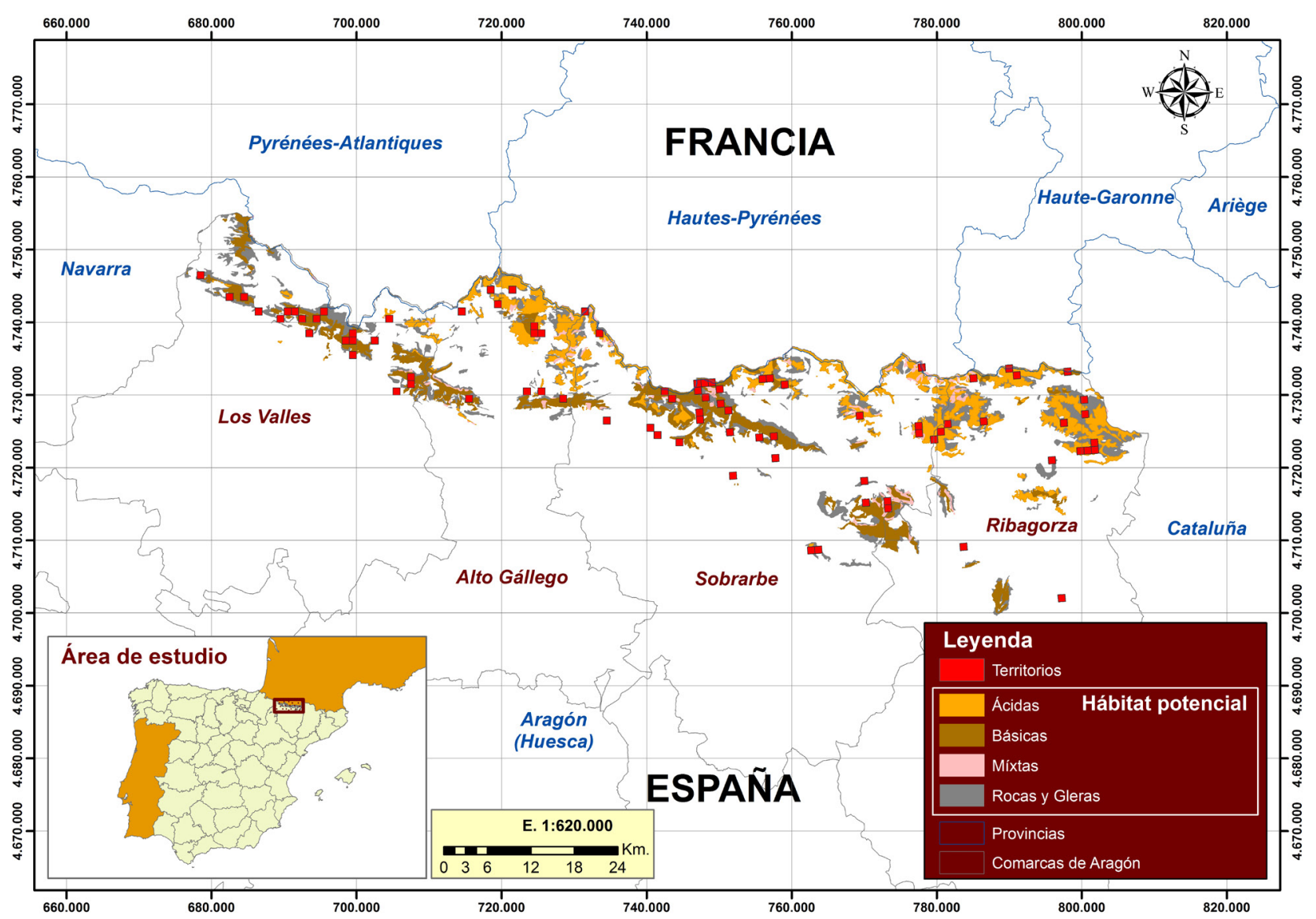

Figura 4: Hábitat potencial del treparriscos (Tichodroma muraria) en Aragón.

Figure 4: Habitat potential Wallcreeper in Aragon.

del área de máxima ocupación (Tabla 4). En los ENP la mayor densidad de territorios es en los Valles (Tabla 5).

\subsection{Estado de conservación}

Según el Libro Rojo de las Aves de España, el treparriscos se considera un taxón No Evaluado (Madroño et al., 2004). Según la UICN (2012) a nivel global el treparriscos se encuentra en una categoría de Preocupación Menor (LC), ya que habiendo sido evaluado no cumple ninguno de los criterios que definen las categorías de En Peligro Crítico, En Peligro Vulnerable o Casi Amenazado (BirdLife International, 2014). Con la información disponible de la especie en Aragón (1.410 individuos reproduc- tores), debería estar incluida en la categoría de Preocupación Menor.

\section{Discusión}

\subsection{Fenología de la reproducción y hábitat}

Según Hernández et al., (1993a) la construcción del nido tiene lugar entre el 21 mayo y el 9 de junio. La incubación solo es realizada por la hembra y dura entre 18,5 y 20 días, iniciándose con la puesta del penúltimo huevo (Glutz von Blotzheim \& Bauer, 1993; Cramp \& Perrins, 1993). Según la literatura se observan pollos en el nido entre el 3 de julio y el 13 de agosto, grupos familiares entre el

Tabla 4: Estimación de densidad en área potencial, máxima ocupación y supra forestal en Aragón.

Table 4: Estimated potential area density, maximum and forestry supra Aragon.

\begin{tabular}{|l|c|c|}
\hline \multicolumn{1}{|c|}{ Estimación } & Área $\left.\mathbf{( K m}^{\mathbf{2}}\right)$ & Densidad (territorios $\mathbf{K m}^{\mathbf{2}}$ ) \\
\hline Área potencial & 664,96 & 0,13 \\
\hline Área supraforestal & $1.491,58$ & 0,06 \\
\hline Área máxima ocupación & 495,45 & 0,18 \\
\hline
\end{tabular}




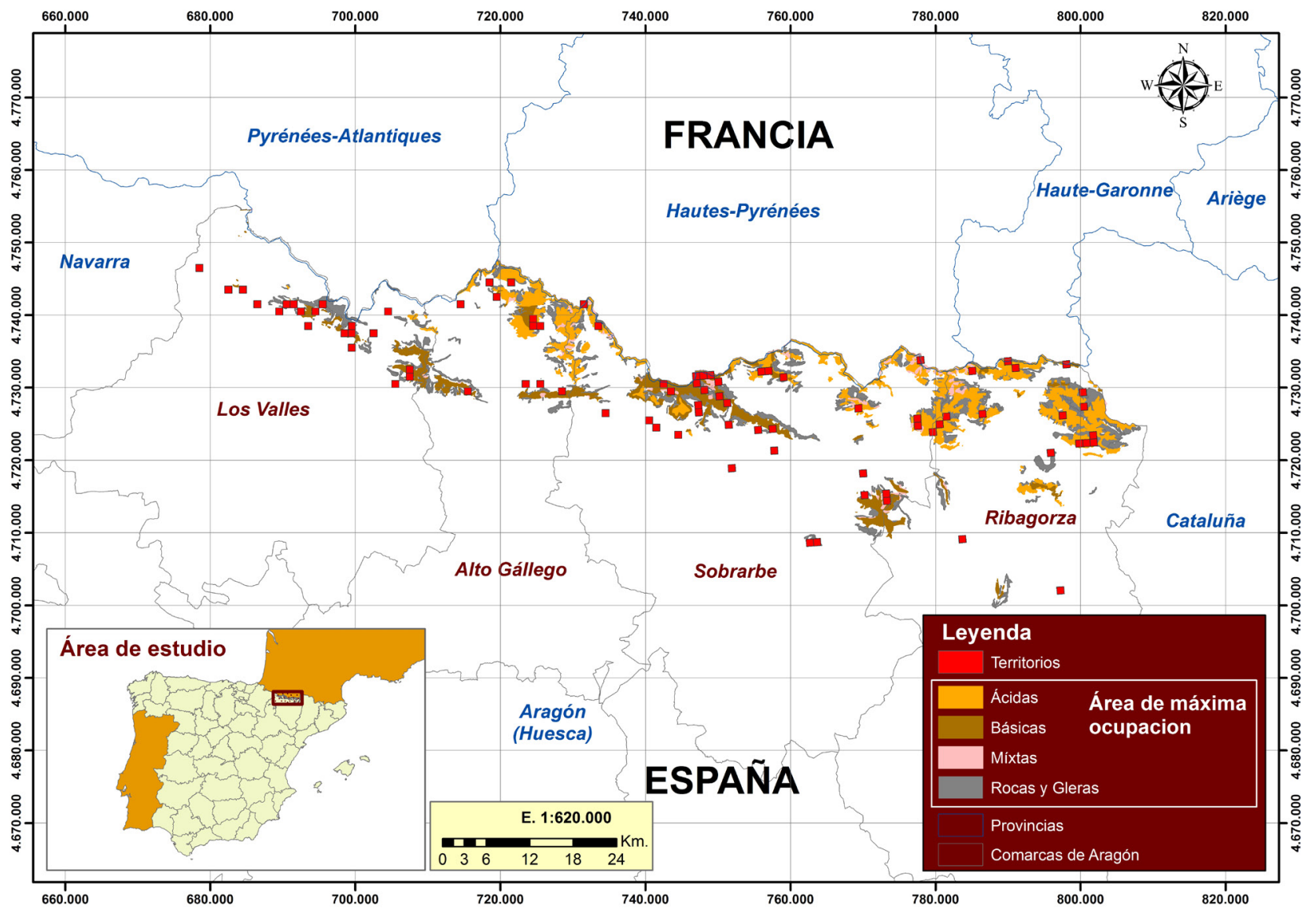

Figura 5. Hábitat de máxima ocupación del treparriscos (Tichodroma muraria) en Aragón.

Figure 5: Habitat maximum occupancy of Wallcreeper in Aragon.

Tabla 5: Estimación de densidad por ENP.

Table 5: Estimated density ENP.

\begin{tabular}{|c|c|c|c|c|}
\hline ENP & $\begin{array}{c}\text { Área hábitat } \\
\text { potencial }\left(\mathbf{K m}^{2}\right)\end{array}$ & $\begin{array}{c}\text { Densidad } \\
\text { (territorios } \mathbf{K m}^{2} \text { ) }\end{array}$ & $\begin{array}{c}\text { Área máxima } \\
\text { ocupación }\left(\mathrm{Km}^{2}\right)\end{array}$ & $\begin{array}{c}\text { Densidad } \\
\text { (territorios } \mathbf{K m}^{2} \text { ) }\end{array}$ \\
\hline Los Valles & 74,1 & 0,21 & 19,16 & 0,8 \\
\hline PNOMP & 66,11 & 0,35 & 55,43 & 0,42 \\
\hline Posets-Maladeta & 180,52 & 0,09 & 172,35 & 0,1 \\
\hline
\end{tabular}

8 y el 17 de agosto y jóvenes independizados entre el 26 de julio y el 21 de septiembre (Hernández et al., 1993a). Los jóvenes son alimentados y criados por ambos sexos. Se hacen volanderos a los 26-30 días y se independizan de sus progenitores cinco a doce días más tarde (Glutz von Blotzheim \& Bauer, 1993; Cramp \& Perrins, 1993; Saniga, 2004). Los datos obtenidos en el presente trabajo sobre la fenología de la reproducción del treparriscos es similar a los obtenidos por los anteriores autores: construcción del nido entre mayo y principios de junio, incubación entre junio y julio, pollos en nido entre junio y finales de julio y grupos familiares en agosto. Los sectores de cría en el Pirineo aragonés se sitúan en un rango altitudinal similar a lo obtenido por otros autores: Báguena et al. (1998), Wouter- sen \& Grasa (2002), Desaulnay (1997) y Hernández et al. (1993b). Esta tendencia de ocupar para la cría zonas de alta montaña se observa en otras montañas meridionales y de clima continental como en Grecia (1.900-2.400 m.s.n.m.), Turquía (2.300-3.100 m.s.n.m.) y Tien-Shan (3.000-3.500 m.s.n.m.) (Löhrl \& Wilson, 2008). Mientras que en montañas septentrionales: Alpes (Suiza: intervalo 350/3.450 m.s.n.m.), Carpatos: intervalo 500/1.800 m.s.n.m., o de clima oceánico como la Cordillera Cantábrica (altitud media 1.680 m.s.n.m.) tiende a ocupar altitudes inferiores (Löhrl \& Wilson, 2008; Saniga, 2004; Hernández et al., 1993b). Se produce un ascenso altitudinal de la especie en agosto, tal y como expone Hernández et al. (1993b) llegándose a observar por encima de los 3.000 m.s.n.m. en Pirineos. 
También se ha llegado a observar en otras Cordilleras a mayor altitud como en Pamir a 4.700 m.s.n.m. (Löhrl \& Wilson, 2008). En el Pirineo oriental (Cataluña) las localidades de reproducción a baja altitud (Collegats: Noguera Pallaresa, 600 m.s.n.m.) son consideradas como irregulares y ocasionales (Aymerich et al., 2012), mientras que en el Pirineo aragonés se reproduce con regularidad en algunas localidades de baja altitud: Congosto de Ventamillo (valle del Esera) y Boca del Infierno (valle de Hecho). La reproducción en valles fluviales a baja altitud es frecuente en Alpes, considerándose la existencia de agua corriente como un factor determinante (Lörhrl, 1976). Según Hernández et al., 1993b, el tipo de hábitat principal del treparriscos durante la época de cría en Pirineos es el roquedo interior no fluvial (85,1\%). Santandreu et al., 2004 expone que las principales áreas de la especie en Cataluña coinciden con importantes afloramientos de zonas calcáreas y graníticas de alta montaña, mientras que en las áreas de esquistos no se detecta su presencia. Según Antor (1995), la especie está notablemente especializada en la explotación de determinados microhábitats: riscos y peñas $(60 \%)$, derrubios de ladera $(40 \%)$ y parches de nieve y sus bordes (10\%). La falta de coincidencia con el resto de las especies alpinas puede ser debida a que estos medios no pueden ser explotados eficientemente por éstas, ya que carecen de la morfología adecuada (Antor, 2002).

\subsection{Distribución}

Distribución continua, presente en todo el Pirineo Axial y Sierras Interiores del Pirineo central español, contactando con las poblaciones occidentales (RoncalLarra/Navarra) (Elosegui, 1985) y orientales (BessiberriAlta Ribagorza/Lérida) y las poblaciones de la vertiente norte del Pirineo (Francia): regiones de Midi-Pyrénées (Nature Midi-Pyrénées, 2011) y Aquitania (http://www. faune-aquitaine.org/). Se amplía en 10 cuadrículas de $10 \times 10 \mathrm{~km}$ la distribución conocida con respecto a la obtenida en el Atlas 1987-1991 (Baguena et al., 1998). Queda por localizar su presencia en dos macizos (Culfreda y Acherito-Mesa de los Tres Reyes) donde existe hábitat potencial para la especie y que precisaría de prospecciones especiales para constatar su presencia, debido a la dificultad que entraña su localización durante la época de reproducción, ya sea por contactos visuales o auditivos mediante la utilización de reclamos. Según Lörhrl \& Wilson (2008) los tipos de roca no influyen en la distribución del Treparriscos, sino la estructura de los cortados. Aymerich et al. (2012) exponen que la distribución de la especie es más continua en sectores con afloramientos calizos y graníticos, que en zonas de esquistos y concluyen que la litología no determina la distribución, pero sí condiciona la abundancia local o densidades a escalas pequeñas, mostrando claras diferencias en la extensión de los territorios según se sitúen en zonas calcáreas o graníticas. También indican que las zonas calcáreas poseen hábitats de mejor calidad y mayor capacidad de soportar poblaciones locales más densas, coincidiendo con otros autores por la preferencia de la especie por las zonas calcáreas (Hernández et al., 1993b; Saniga, 2004). Es posible que también intervengan otros factores como la altitud, orientación, superficie vertical de los cortados, rocosos (Aymerich et al., 2012).

\subsection{Abundancia y estima del tamaño poblacional}

La estimación de densidad del Pirineo aragonés, considerando todo el conjunto de área supra forestal, es muy similar a la obtenida en el Pirineo oriental (Cataluña): 0,05-0,07 parejas/ $\mathrm{Km}^{2}$ (Aymerich et al., 2012) y a zonas de los Alpes: 0,05-0,06 parejas/ $\mathrm{Km}^{2}$ Alpes-Baviera (área de $635 \mathrm{Km}^{2}$ ), 0,07-0,09 parejas/ $\mathrm{Km}^{2}$ Alpes-Bearneses (área de $2.895 \mathrm{Km}^{2}$ ), 0,03 parejas/ $\mathrm{Km}^{2}$ Alpes-Tirol (área de $320 \mathrm{Km}^{2}$ ), 0,01-0,02 parejas/ $\mathrm{Km}^{2}$ Alpes-Brescia (área de $4.749 \mathrm{Km}^{2}$ ) (Löhrl \& Wilson, 2008). La estimación de densidad del Pirineo aragonés, considerando tan solo el área potencial, es inferior a lo obtenido en Cataluña: 0,260,33 parejas $/ \mathrm{Km}^{2}$. En estimas de densidad realizadas a pequeña escala en el Pirineo catalán $\left(0,1-0,6\right.$ parejas $\left./ \mathrm{Km}^{2}\right)$ y Picos de Europa $\left(0,6-0,7\right.$ parejas/ $\left.\mathrm{Km}^{2}, 4-9 \mathrm{Km}^{2}\right)($ GIALeón, 2012) las densidades son menores. Si la población del Pirineo catalán es de 65 territorios, la del Pirineo aragonés para el área de máxima ocupación sería de 525 y para todo el área potencial de 704 territorios; en el Pirineo navarro existen tan solo registros en el alto valle del Roncal (Elosegui, 1985), la población de la vertiente sur de los Pirineos (España) se podría estimar entre 595-772 territorios potenciales. Esto supondría que las primeras estimas para la población española de la especie estarían muy sobrestimadas: 9.000-12.000 territorios (Hernández, 1997), pero que serían similares a las expuestas por Aymerich et al. (2012): entre 500 y 1.000 parejas. Al igual que expone Aymerich et al. (2012) los resultados obtenidos en el presente artículo, exigirían recalcular las estimas realizadas para la población española de Treparriscos. Además cabría revisar la estima realizada por Hernández (1997), que fue utilizada para las estimas de la población europea (BirdLife Internacional/EBCC, 2000; BirdLife Internacional, 2004). Para ello sería necesario además conocer el tamaño de la población reproductora de la Cordillera Cantábrica.

\subsection{Estado de conservación}

En Europa se considera que el treparriscos posee un estado de conservación favorable (BirdLife Internacional, 2004). En Aragón el estado de conservación de la especie también parece favorable, al igual que en la población oriental (Cataluña) (Aymerich et al., 2012), ya que sus hábitats no han sufrido impactos negativos, ni se han visto afectados por actividades humanas. La única amenaza potencial podría ser el calentamiento global que podría producir contracciones en su distribución actual (Araujo et al., 2011). A diferencia de la población del Pirineo catalán, que ha sido catalogada como En Peligro, la pobla- 
ción del Pirineo aragonés se ha incluido en la categoría de Preocupación Menor, con una distribución continua y un número de ejemplares reproductores por encima de la categoría de Vulnerable.

\section{Agradecimientos}

A todo el equipo de la FCQ y muy especialmente a los colaboradores que han enviado información: Juan Carlos Ascaso, Jesús Lavedán, Rafael Vidaller, José Antonio Villacamapa, José Manuel Sánchez, Julio Guiral, Fernando Tallada, Eduardo Viñuales, Joaquín Guerrero, Juan Carlos Albero, Luis Palacio, Javier Sampietro, Luis Gracia, Carlos Usieto, Ramón Antor y Pascual López-López.

\section{Referencias}

Araujo, M., Guilhaumon, F., Pozo, I. \& Gómez R., 2011. Impactos, vulnerabilidad y adaptación de la biodiversidad española frente al cambio climático. Volumen 2: Fauna de vertebrados. Ministerio de Medio Ambiente y Medio Rural y Marino, Museo Nacional de Ciencias Naturales y CSIC.

Antor, R., 1995. The importance of arthropod fallout on snow patches for the foraging of high-alpine birds. Journal of Avian Biology, 26(1): 81-85. http://dx.doi.org/10.2307/3677216.

Antor, R. 2002. Ecología de las comunidades de paseriformes alpinos en el Pirineo. Consejo de Protección de la Naturaleza de Aragón, 174, Zaragoza.

Asso, I., 1784. Introductio in Oryctographiam et Zoologiam Aragoniae. Kessinger Pub Co: 208.

Atlas des Oiseaux Nicheurs Aquitania 2014. Disponible en: http://www.faune-aquitaine.org/index.php?m id=505.

Aymerich, P., Capdevila, F., Canut, J., Roig, J. \& Santandreu, J., 2012. Distribuciò I abundància de la població reproductora de Pela-roques Tichodroma muraria a Catalunya. Revista Catalana d'Ornitologia, 28: 1-19.

Báguena, G., Díez, O, Gil, J.A. \& Lorente, L., 1998. Treparriscos. Tichodroma muraria. Pp. 374-375. En: Sampietro, F. J., Pelayo, E., Hernández, F., Cabrera, M., Guiral, J. (Eds). Aves de Aragón. Atlas de especies nidificantes. Diputación General de Aragón e Ibercaja, 568, Zaragoza.

BirdLife International 2000/European Bird Census Council. European bird populations: estimates and trends. BirdLife International Conservation Cambridge UK: BirdLife International (BirdLife Conservation Series No. 10).

BirdLife International 2004. Birds in the European Union: a status assessment. Wageningen, The Netherlands: BirdLife International.

BirdLife International 2014. Tichodroma muraria. IUCN Red List of Threatened Species. Version 2014.1. (www.iucnredlist.org).

Cramp, S. \& Perrins, C. M., 1993. Handbook of the Birds of Europe, the Middle East and North Africa. The Birds of the Western Palearctic. Volume VII. Flycatchers to Shrikes. Oxford University Press, London.

Desaulnay, P., 1997. Tichodrome echelette (Tichodroma muraria). In: Joachim, J., Bouquet, J.F. \& Faure, C.: Atlas des oiseaux nicheurs de Midi-Pyrénées. Annees 1985 a 1989. Pp. 209-201. Toulouse: Association Regionale Ornithologique de Midi-Pyrénées.

Elosegui, J., 1985. Navarra. Atlas de aves nidificantes (19821984). Treparriscos Tichodroma muraria. Pp. 172. Caja de Ahorros de Navarra.
GIA (Grupo Ibérico de Anillamiento)-León 2012. Situación del Treparriscos (Tichodroma muraria) en el Macizo Central de Picos de Europa. Informe inédito.

Glutz von Blotzheim, U.N. \& Bauer, K.M., 1993. Handbuch der Vögel Mitteleuropas. Band 13/II. Passeriformes (4. Teil). Sittidae-Laniidae. Aula Verlag, Wiesbaden.

Hagemeijer, E.J. \& Blair, M.J., 1997. The EBBC atlas of European Breeding Birds: Their distribution an abundance. London: EBCC.

Hernández, A., 1997. Treparriscos Tichodroma muraria. Pp. 456-457. En: Purroy, F. J. (Coord.). Atlas de las aves de España (1975-1995). SEO-BirdLife-Lynx Edicions, Barcelona.

Hernández, A., Alegre, J., Velasco, T. \& Casas, V.M., 1993a. Fenología de reproducción del Treparriscos Tichodroma muraria en la Península Ibérica. Butlletí Grup Catalá d'Anellament, 10: 47-49.

Hernández, A., Alegre, J., Velasco, T. \& Casas, V.M., 1993 b. Hábitat del Treparriscos Tichodroma muraria en la Península Ibérica e Islas Baleares. Butlletí Grup Catalá d'Anellament, 10: 39-45.

Hernández, A., Hauri, R. \& Saniga, M., 1997. Wallcreeper. Tichodroma muraria. En: Hagemeijer, J.M. \& Blair, M.J., (Eds). The EBCC Atlas of European Breeding Birds: Their Distribution and Abundance. London: EBCC.

Herrero, A., 2003. Treparriscos. Tichodroma muraria. Pp. 520521. En: Martí, T., del Moral, J.C. (Eds): Atlas de las Aves Reproductoras de España. Dirección General de Conservación de la Naturaleza-Sociedad Española de Ornitología, Madrid.

Lörhrl, H., 1976. Der Mauerlüfer. Die Neue Brehm-Bücherei 498. A. Ziemsen. Wittenberg Lutherstadt.

Löhrl, H. \& Wilson, M.G., 2008. Family Tichodromidae (Wallcreeper). Pp. 146-165. In: Del Hoyo, J., Elliot, A., Christie, D. (Eds.). Handbook of the Birds of the World. Volume 13. Penduline-tits to Shrikes. Lynx Edicions, Barcelona.

Nature Midi-Pyrénées. 2011. Atlas des oiseaux nicheurs de Midi-Pyrénées (disponible en: http://www.naturemp.org/ATLAS ORNITHO/BIB avanc.php).

Madroño, A., González, C \& Atienza, J.C., 2004. Libro Rojo de las Aves en España. Dirección General para la Biodiversidad. SEO/BirdLife. Madrid.

Pedrocchi, C., Moreno, D. \& Cervantes, J., 2007. Comunidades de aves nidificantes en pastos supraforestales pirenaicos. $\mathrm{Su}$ evolución a lo largo del año. Pirineos, 162: 109-123.

Santandreu, J. Carulla, A. Garcia-Petit, J \& Aymerich, P., 2004. Pela-roques Tichodroma muraria. In: Estrada, J., Pedrocchi, V., Brotons, L. \& Herrando, S. (Eds.) Atles dels ocells nidificants de Catalunya 1999-2002. Pp. 446-467-Institut Catala d'Ornitologia (ICO)/Lynx Edicions, Barcelona.

Saniga, M., 2004. Features of the Wallcreeper Tichodroma muraria breeding habitat in the Wets Carpathians. Monticola, 95 (9): 222-227.

Sanz-Zuasti, J. \& Velasco T., 1999. Guía de las Aves de Castilla y León. Náyade. Medina del Campo, 200, Valladolid.

UICN (2012). Categorías y Criterios de la Lista Roja de la UICN: Versión 3.1. Segunda edición. Gland, Suiza y Cambridge, Reino Unido: UICN. vi+34pp. Originalmente publicado como IUCN Red List Categories and Criteria: Version 3.1.

Wallis, H.M., 1895. Notes on the birds of the central Pyrenees. Ibis, 37: 64-85. http://dx.doi.org/10.1111/j.1474-919X.1895. tb06510.x.

Woutersen, K. \& Grasa, M., 2002. Parque Nacional de Ordesa y Monte. Perdido. Atlas de las Aves. Kees Woutersen publicaciones, 195, Huesca. 